\title{
Antinociceptive and Immune Effects of Delta-9-Tetrahydrocannabinol or Cannabidiol in Male Versus Female Rats with Persistent Inflammatory Pain $\$$
}

\author{
Stevie C. Britch, (D)Alan G. Goodman, Jenny L. Wiley, Abby M. Pondelick, \\ and Rebecca M. Craft \\ Center on Drug and Alcohol Research, Department of Behavioral Science, University of Kentucky, Lexington, Kentucky (S.C.B.); \\ School of Molecular Biosciences, Paul G. Allen School for Global Animal Health (A.G.G.), Integrative Physiology and \\ Neuroscience (A.M.P.), and Department of Psychology (R.M.C.), Washington State University, Pullman, Washington; and RTI \\ International, Research Triangle Park, North Carolina (J.L.W.)
}

Received October 17, 2019; accepted March 11, 2020

\begin{abstract}
Chronic pain is the most common reason reported for using medical cannabis. The goal of this research was to determine whether the two primary phytocannabinoids, delta-9-tetrahydrocannabinol (THC) and cannabidiol (CBD), are effective treatments for persistent inflammatory pain. In experiment 1 , inflammation was induced by intraplantar injection of Complete Freund's adjuvant (CFA). Then THC $(0.0-4.0 \mathrm{mg} / \mathrm{kg}$, i.p.) or CBD (0.0-10 mg/kg, i.p.) was administered twice daily for 3 days. On day 4 , THC, CBD, or vehicle was administered, and allodynia, hyperalgesia, weight-bearing, locomotor activity, and hindpaw edema were assessed $0.5-4$ hours postinjection. In experiment 2, CFA or mineral oil (no-pain control)-treated rats were given THC (2.0 mg/kg, i.p.), CBD (10 mg/kg, i.p.), or vehicle in the same manner as in experiment 1 . Four hours postinjection on day 4 , serum samples were taken for analysis of cytokines known to influence inflammatory pain: interleukin (IL)- $1 \beta$, IL-6, IL-10, interferon (IFN)- $\gamma$, and tumor necrosis factor (TNF)- $\alpha$. THC dose-dependently reduced pain-related behaviors but did not reduce hindpaw edema, and little tolerance developed to THC's effects. In contrast, CBD effects on inflammatory pain were
\end{abstract}

minimal. THC produced little to no change in serum cytokines, whereas CBD decreased IL- $1 \beta$, IL-10, and IFN- $\gamma$ and increased IL-6. Few sex differences in antinociception or immune modulation were observed with either drug, but CFA-induced immune activation was significantly greater in males than females. These results suggest that $\mathrm{THC}$ may be more beneficial than $\mathrm{CBD}$ for reducing inflammatory pain in that THC maintains its efficacy with short-term treatment in both sexes and does not induce immune activation.

\section{SIGNIFICANCE STATEMENT}

The pain-relieving effects of cannabidiol (CBD) and delta-9tetrahydrocannabinol (THC) are examined in male and female rats with persistent inflammatory pain to determine whether individual phytocannabinoids could be a viable treatment for men and women with chronic inflammatory pain. Additionally, sex differences in the immune response to an adjuvant and to THC and CBD are characterized to provide preliminary insight into immune-related effects of cannabinoid-based therapy for pain.

\section{Introduction}

People across the United States use medical cannabis to treat chronic pain, including inflammation-related pain (Baron et al., 2018). Clinical trials suggest that cannabinoids are weak analgesics compared with Food and Drug Administration-approved analgesics, such as opioids (Stockings et al., 2018). It is unclear to what extent each of the two primary phytocannabinoids, delta-9-tetryhydrocannabinol

This work was funded by the National Institute on Drug Abuse [Grants DA016644, T32DA035200]. The content is solely the responsibility of the authors and does not necessarily represent the official views of the National Institutes of Health.

Laboratory of origin: Rebecca M. Craft.

https://doi.org/10.1124/jpet.119.263319.

S This article has supplemental material available at jpet.aspetjournals.org.
(THC) and cannabidiol (CBD) (Mechoulam et al., 1976; Hanuš et al., 2016), contribute to cannabis-induced analgesia during inflammation and whether these cannabinoids are equally effective in both sexes. In rats, THC reduced mechanical hyperalgesia in males with adjuvant-induced arthritis (Cox and Welch, 2004) and reduced mechanical allodynia, heat hyperalgesia, and edema associated with Complete Freund's adjuvant (CFA)-induced hindpaw inflammation in both sexes (Craft et al., 2013), suggesting that acutely administered THC is effective against inflammatory pain in both sexes.

CBD also reduced pain in rodent models of inflammation. Acute CBD reduced hyperalgesia and edema in male rats with carrageenan-induced hindpaw inflammation (Costa et al., 2004), and once-daily CBD for 7 days reversed CFA-induced hyperalgesia (Costa et al., 2007). Acute CBD reduced carrageenan-induced hyperalgesia but not paw edema in

ABBREVIATIONS: \% BL, \% of baseline; CB, cannabinoid; CBD, cannabidiol; CFA, Complete Freund's adjuvant; IFN, interferon; IL, interleukin; LPS, lipopolysaccharide; THC, delta-9-tetrahydrocannabinol; TNF, tumor necrosis factor. 
another study (Rock et al., 2018). CBD reduced allodynia and edema associated with zymosan A-induced hindpaw inflammation in female mice (Gallily et al., 2015, 2018). Finally, CBD reduced joint hyperalgesia and edema in male rats with knee arthritis (Hammell et al., 2016) and improved clinical scores in male mice with arthritis (Malfait et al., 2000). Although females and males have not been directly compared, these studies suggest that CBD alleviates inflammatory pain in both sexes.

Despite well-documented sex differences in humans with pain (Unruh, 1996; Blyth et al., 2001; Tighe et al., 2014), few studies have investigated sex differences in cannabinoid analgesia, and these have shown mixed results (Redmond et al., 2008; Cooper and Haney, 2014, 2016). In animal studies, our laboratory has shown that THC is more potent in female rats compared with male rats using acute pain tests (Tseng and Craft, 2001; Craft et al., 2012). However, females develop greater tolerance than males to repeatedly administered THC; thus, sex differences wane when THC is administered repeatedly to healthy rats (Wakley et al., 2014, 2015; Greene et al., 2018). No studies have compared the development of tolerance to THC's (or CBD's) pain-relieving effects in male versus female rats with inflammatory pain.

Cannabinoids produce analgesia via multiple neural mechanisms, including actions at type 1 and type 2 cannabinoid (CB), CB1 and CB2, receptors and transient receptor potential vanilloid type 1 receptors located centrally and peripherally (Starowicz and Finn, 2017; Guerrero-Alba et al., 2019) and also may decrease pain via immunosuppression (Klein and Cabral, 2006; Katchan et al., 2016). CB2 receptors are located on immune cells throughout the body, including B cells, natural killer cells, monocytes, neutrophils, and $\mathrm{T}$ cells (Galiègue et al., 1995); CB1 (Katchan et al., 2016) and transient receptor potential vanilloid type 1 receptors also have been identified on immune cells (Kong et al., 2017). THC reduced cytokines, such as tumor necrosis factor (TNF)- $\alpha$, interleukin (IL)-1 $\beta$, and IL-6 in rat microglia (Puffenbarger et al., 2000), and the synthetic cannabinoids WIN55,212-2 and HU-210 decreased serum TNF- $\alpha$ and interferon (IFN)- $\gamma$ but increased serum IL-10 in endotoxemic male mice (Smith et al., 2000). CBD decreased IFN- $\gamma$ in draining lymph nodes, decreased TNF- $\alpha$ release from synovial cells of arthritic male mice, and inhibited lipopolysaccharide (LPS)-induced increases in serum TNF- $\alpha$ in female mice (Malfait et al., 2000). Finally, CBD reduced serum TNF- $\alpha$ in a zymosan A-induced hindpaw inflammation model (Gallily et al., 2015, 2018). These studies suggest that changes in cytokines could contribute to cannabinoid analgesia.

Considering the role of the immune system in chronic inflammatory pain (Raoof et al., 2018) and the greater prevalence of chronic inflammatory pain in women than men (Unruh, 1996), research investigating sex differences in cytokines during chronic pain is needed. Sex differences in cytokine levels have been reported but may be cell- and adjuvant-specific (reviewed in Klein and Flanagan (2016)). No studies have investigated sex differences in the immune response to CFA, even though CFA is commonly used to induce inflammatory pain in rodents.

Given THC's and CBD's antinociceptive effects in male rodents with inflammatory pain and sex differences in THCinduced antinociception and tolerance to THC, in this study we compared the antinociceptive and antiedematous effects of acute versus repeated administration of THC or CBD in male versus female rats with persistent inflammatory pain. We also provide a preliminary characterization of sex differences in cytokine responses to CFA, THC, and CBD.

\section{Methods}

Animals. All experiments were completed in accordance with the National Institutes of Health Guide for the Care and Use of Laboratory Animals (Institute of Laboratory Animal Resources, 2011). Male and female Sprague-Dawley rats aged 60-90 days were used (bred inhouse from Envigo stock, Livermore, CA). They were housed in samesex groups of 2 to 3 under a 12:12-hour light:dark cycle (lights on at 0700 hours). Each home cage contained soft, absorbant bedding made from wood pulp (TEK-Fresh; Envigo) and a 12-15-cm long $\times 10-\mathrm{cm}$ diameter PVC tube. The room was maintained at $21 \pm 2{ }^{\circ} \mathrm{C}$ with approximately 25\% humidity. Food (LabDiet 5001; Animal Specialties \& Provisions, Quakertown, PA) and water were available ad libitum except during testing. Rats were randomly assigned to treatment groups with the exception that same-sex siblings generally were not assigned to the same treatment group.

Drugs. Drugs were obtained from the National Institute on Drug Abuse (THC and CBD; Bethesda, MD) and from Cayman Chemical Co. (CBD; Ann Arbor, MI). THC and CBD were dissolved in a 1:1:18 ethanol:cremophor:saline solution, which also served as the vehicle. Drugs were administered by intraperitoneal injection in volumes of $1 \mathrm{ml} / \mathrm{kg}$. THC doses were $0.5,1.0,2.0$, or $4.0 \mathrm{mg} / \mathrm{kg}$, and CBD doses were $1.25,2.5,5.0$, or $10 \mathrm{mg} / \mathrm{kg}$. Experimenters were blind to dose. CFA ( $1 \mathrm{mg} / \mathrm{ml}$ heat-killed Mycobacterium tuberculosis) was purchased from Millipore Sigma (St. Louis, MO) and administered via intraplantar injection to the right hindpaw.

Apparatus. Mechanical sensitivity was assessed using an electronic von Frey aesthesiometer (IITC Inc., Woodland Hills, CA). Thermal sensitivity was assessed using a Hargreaves apparatus (Ugo Basile Plantar Test, model 7371; Collegeville, PA). Hindpaw weight-bearing was measured using an incapacitance meter (Columbus Instruments, Columbus, $\mathrm{OH}$ ). Horizontal locomotor activity was measured using a photobeam apparatus (Opto-varimex; Columbus Instruments): 15 photobeams cross the width of a $20 \times 40 \times 23$-cm clear plastic rodent cage, with photobeams spaced $2.5 \mathrm{~cm}$ apart and $6.5 \mathrm{~cm}$ high. Paw edema was quantified by measuring maximal dorsalventral hindpaw thickness with calipers.

Behavioral Procedures. Figure 1 shows a timeline of behavioral procedures. On day 1 , starting at 0800 hours, rats $(n=8-12 / \mathrm{sex} /$ treatment group) were weighed, and baseline measurements were taken. First, rats were placed in hanging wire cages to habituate for approximately 20 minutes. The threshold at which the rat responded when the von Frey probe was applied to the plantar surface of the right hindpaw was recorded in grams. Three assessments were made over approximately 2 minutes with approximately 30 seconds between tests. The Hargreaves test was completed next; latency to withdraw the right hindpaw was recorded to the nearest 0.1 second with a cutoff of 31 seconds. Three assessments were made over approximately 2 minutes with approximately 30 seconds between tests. Next, maximal dorsal-ventral thickness of the right hindpaw was measured in millimeters. Rats were then placed in a standing position into a plastic chamber, and weight-bearing (in grams) on each hindpaw was recorded after 15 seconds, three times over approximately 1 minute. Finally, rats were placed into locomotor chambers, and the number of photobeam breaks in 10 minutes was recorded. Immediately after baseline measures were taken, rats were briefly anesthetized with isoflurane, and $0.1 \mathrm{ml}$ CFA was injected into the plantar surface of the right hindpaw. One hour after CFA injection, vehicle or a single dose of THC or CBD was injected intraperitoneally (at approximately 1000 hours). The same drug/dose was injected again at 1700 hours that day and on days 2 and 3 at approximately 0800 and 1700 hours; rats were weighed daily prior to the morning injection. 
On day 4 at 0800 hours, rats that had received THC or CBD received another injection of the same drug and dose, whereas those that had received vehicle received a dose of THC, CBD, or vehicle. At 30, 60, 120, and 240 minutes postinjection, von Frey, Hargreaves, weightbearing, and locomotor tests were conducted as described for day 1 . Paw thickness was also measured on day 4, at the 240-minute time point only. To determine whether drug effects persisted after termination of treatment, rats were tested on all assays again on day 8 , but no injections were given on days 5-8.

Immunologic Procedures. Separate rats were used to assess immunologic effects of THC and CBD. On day 1 at 0800 hours, rats $(n=$ 8/sex/treatment group) were injected in the right hindpaw with $0.1 \mathrm{ml}$ of CFA or mineral oil (non-inflamed control). One hour postinjection, THC $(2.0 \mathrm{mg} / \mathrm{kg})$, CBD $(10.0 \mathrm{mg} / \mathrm{kg})$, or vehicle was injected intraperitoneally; twice-daily drug administration followed the same timeline as described above (Behavioral Procedures), and these doses were chosen based on results from the first experiment (THC $2 \mathrm{mg} / \mathrm{kg}$ reduced multiple pain-related behaviors; CBD $10 \mathrm{mg} / \mathrm{kg}$ reduced paw edema). Four hours postinjection on day 4 (to align with the time at which the final behavioral tests were conducted, and edema was measured in the first experiment), rats were euthanized; trunk blood and spleen were collected. Spleens were immediately weighed as a measure of overall toxicity and immune response (Bronte and Pittet, 2013). Blood samples were centrifuged for 10 minutes at $2000 \mathrm{rpm}$ and $20^{\circ} \mathrm{C}$, and then serum supernatant was collected; serum samples were flash frozen in liquid nitrogen and stored at $-80^{\circ} \mathrm{C}$ for later determination of inflammatory cytokine concentration. Serum was analyzed for TNF- $\alpha$, IL-1 $\beta$, IL-6, IL-10, and IFN- $\gamma$ using ELISAs (Thermo Fisher Scientific, Waltham, MA), according to the manufacturer's instructions.

Data Analysis. The mean of the three trials conducted for each assay (von Frey, Hargreaves, and weight-bearing) was calculated to yield single baseline and drug test scores at each time point for each rat. On the weight-bearing test, only data for the right (CFA-injected) hindpaw were used. Data were analyzed using SPSS Statistics for Windows, Version 25.0. Baseline values (on each assay) were compared on each assay using a one-way ANOVA to determine whether there were sex differences. THC and CBD were tested at different times with different vehicle control groups (THC data were collected first over 28 months, and immediately thereafter CBD data were collected over 19 months), therefore baseline values between studies were also compared on each assay using a one-way ANOVA to determine whether baselines differed between CBD and THC experiments. Because there were baseline differences between experiments, analyses of THC and CBD effects were conducted separately. A priori power analysis was conducted using $\mathrm{G}^{*}$ power3 to determine sample size for each study (behavior-THC, behavior-CBD, and immune) (Faul et al., 2007).

Von Frey, Hargreaves, weight-bearing, locomotor, and pawthickness drug test scores were each transformed to \% of baseline [(drug score/baseline score $) \times 100]$ for each rat to adjust for sex differences and other individual differences in baselines (see Results). Missing baseline locomotor data ( $<1 \%$ of cases) were replaced by the mean baseline for that treatment group.

Although data were not normally distributed, the complexity of the experimental design required the use of parametric analyses. No outliers were identified using Grubb's test (Grubbs, 1969). Percent baseline time course data (von Frey, Hargreaves, weight-bearing, locomotor) from day 4 were then analyzed using a repeated measure, four-way ANOVA, to determine whether the effects produced by either drug (THC dose or CBD dose: five levels) in each sex (sex: two levels) changed over time (time, repeated measure: four levels) and whether these effects differed between rats treated with drug acutely versus repeatedly (chronicity: two levels). THC effects on day 4 were timedependent on only two measures (Hargreaves and weight-bearing), and no CBD effects were time-dependent. Therefore, to simplify data presentation and analysis, the mean of scores at the four time points (30, 60, 120, 240 minutes postinjection) was calculated on von Frey, Hargreaves, and weight-bearing for each rat, and graphs and analyses of these data are presented in the Results section. Thus, three-way ANOVAs were conducted on mean scores and paw-thickness scores at 240 minutes postinjection to determine the effects produced by either drug (THC dose or CBD dose: five levels) in each sex (sex: two levels) and to determine whether these effects differed between rats treated with drug acutely versus repeatedly (chronicity: two levels). Planned comparisons for the THC experiment can be found in Supplemental Table 1 and for the CBD experiment can be found in Supplemental Table 2.

Day 8 data were also analyzed using a three-way ANOVA to determine the effects produced by either drug (THC dose or CBD dose: five levels) in each sex (sex: two levels), and to determine whether these effects differed between rats that had received drug acutely on day 4 versus repeatedly on days 1-4 (chronicity: two levels).

Body weight data were transformed to percent of day 1 body weight (baseline) to adjust for individual differences in initial body weight. Days $2-4$ and 8 transformed body weight data were analyzed using a repeated measure, three-way ANOVA, to determine whether the effects produced by either drug when given repeatedly (THC dose or CBD dose: five levels) in each sex (sex: two levels) changed over time (day, repeated measure: four levels).

To adjust for organ weight differences inherent among rats that differ in body size/weight, spleen weight was transformed to \% body weight for each rat [spleen weight $(g) / b o d y ~ w e i g h t ~(g) \times 100]$. Several serum samples were unquantifiable because of low absorbance below the standard's threshold (3.6\% of samples); these samples were not included in analyses. Transformed spleen weight data and serum cytokine concentrations were each analyzed via three-way ANOVA to determine the effects produced by each treatment condition (vehicle vs. single vs. repeated drug exposure: three levels) in each sex (sex: two levels) and to determine whether these effects differed between

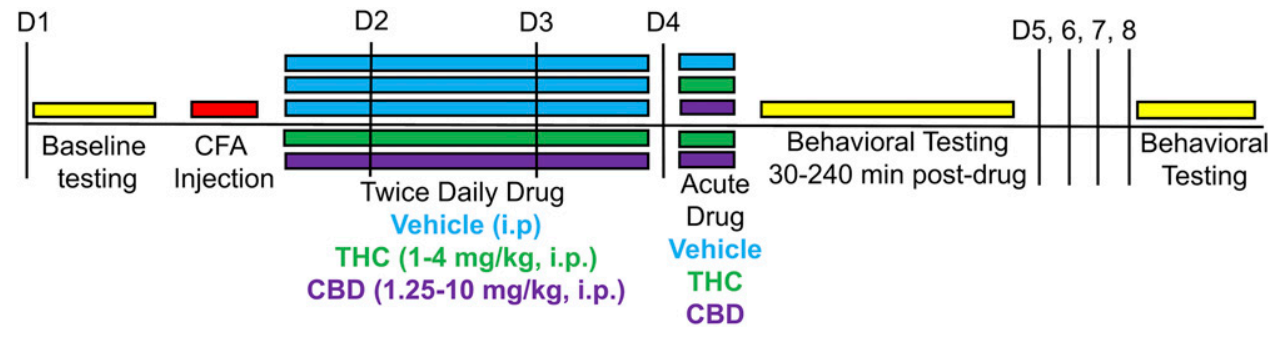

Fig. 1. Timeline for behavioral experiment (Experiment 1). On day 1 (D1), rats were baselined and then injected with CFA in the right hindpaw. One hour postCFA, rats received CBD, THC, or vehicle. Rats received the same injection again at approximately 1700 hours on day 1 and twice daily on days 2 and 3 (D2, D3). On day 4 (D4), rats that had previously received THC or CBD received another injection of the same dose, whereas rats that had previously received vehicle received either another injection of vehicle or an acute injection of THC or CBD. Rats were tested on behavioral assays from 30 to 240 minutes postinjection on day 4 . Rats were tested again on day 8 (D8) but did not receive injections on days 5-8. 
healthy rats versus rats with inflammation (CFA: two levels). To subsequently determine CBD and THC effects in healthy (mineral oil-treated controls) only and in CFA-treated rats only, two-way ANOVAs were conducted (sex: two levels; vehicle vs. single vs. repeated drug exposure: three levels). Dunnett's $t$ test was used for post hoc determination of significance. Significance level was $P \leq 0.05$ for all statistical tests.

\section{Results}

\section{Experiment 1: Acute Versus Repeated THC or CBD Effects on Inflammatory Pain}

Baseline Data. Analysis of baseline data obtained before CFA injection showed that Hargreaves, weight-bearing, and paw-thickness baseline values were lower in the CBD experiment than in the THC experiment (all $P<0.05$ ), perhaps because of slightly smaller size/body weight of rats in the CBD experiment compared with the THC experiment (males: THC experiment $355.2 \pm 3.8 \mathrm{~g}$, CBD experiment $338.9 \pm 2.9 \mathrm{~g}$; females: THC experiment $247.7 \pm 2.7 \mathrm{~g}$, CBD experiment $229.2 \pm 2.1 \mathrm{~g}$ ). There were also sex differences in baselines on all assays in the THC and CBD experiments. Von Frey mechanical thresholds were lower in females than males $\left(60.62 \pm 1.11\right.$ vs. $78.26 \pm 1.11 \mathrm{~g}$, respectively; sex, $\mathrm{F}_{1,332}=$ 127.23, $P<0.001$ ). On the Hargreaves test, paw withdrawal latency was shorter in females than males $(8.78 \pm 0.30 \mathrm{vs} .9 .96$ \pm 0.30 seconds, respectively; sex, $\mathrm{F}_{1,332}=7.76, P=0.006$ ). Females also placed less weight on the right hindpaw compared with males $(84.03 \pm 1.54$ vs. $116.68 \pm 1.54 \mathrm{~g}$, respectively; sex, $\mathrm{F}_{1,332}=223.51, P<0.001$ ). Maximum dorsalventral thickness of the right hindpaw was significantly less at baseline in females compared with males $(4.92 \pm 0.04$ vs. 5.59 $\pm 0.04 \mathrm{~mm}$, respectively; sex, $\mathrm{F}_{1,332}=124.15, P<0.001$ ). Also, females locomoted significantly more than males $(1091 \pm 20$ vs. $1029 \pm 20$ photobeam breaks, respectively; sex, $\mathrm{F}_{1,332}=$ 4.56, $P=0.033$ ). Given these sex differences in baseline measures, all data were transformed to \% of each rat's baseline for each measure before analysis of drug effects.

Mechanical Allodynia. To determine whether THC and CBD decreased pressure-related nociception, mechanical allodynia was measured on day 4 and day 8 (see Supplemental Table 1 for planned comparisons in the THC experiment and Supplemental Table 2 for planned comparisons in the CBD experiment). Figure 2 shows the mean of day 4 time course data on the von Frey test in males and females treated acutely or repeatedly with THC (Fig. 2, A and B) or CBD (Fig. 2, C and D). In the THC experiment, CFA decreased mechanical threshold to a mean of $37.9 \% \pm 6.5 \%$ of baseline (\% BL) in males and $45.8 \% \pm 2.9 \%$ BL in females (see vehicle-treated groups). Compared with vehicle-treated controls, THC increased mechanical threshold (THC, $\mathrm{F}_{4,145}=19.49, P<0.001$ ). Although females had higher \% BL scores than males (sex, $\mathrm{F}_{1,145}=5.16, P=0.025$ ), there were no significant sex differences in THC effect (sex $\times$ THC, $\mathrm{F}_{4,145}=0.77, P=$ 0.550 ). Analysis in only males showed that THC was antiallodynic (THC, $\mathrm{F}_{4,71}=7.70, P<0.001$ ); all doses produced antiallodynia compared with vehicle treatment (all $P \leq 0.010$, Dunnett's), and effects did not differ between males treated with THC acutely versus twice-daily for 3 days (chronicity, $\mathrm{F}_{1,71}=0.041, P=0.841$ ). Analysis in only females showed that THC was antiallodynic compared with vehicle treatment (THC, $\mathrm{F}_{4,74}=14.10, P<0.001$ ); effects of $1.0-4.0 \mathrm{mg} / \mathrm{kg}$ were significant (all $P<0.001$, Dunnett's) and did not differ between females treated with THC acutely versus twicedaily for 3 days (chronicity, $\mathrm{F}_{1,74}=0.63, P=0.430$ ).

On day 8-after several days of no injections-CFA + vehicle-treated rats had decreased mechanical thresholds compared with their baselines: $54.4 \% \pm 6.6 \%$ BL in males and $52.3 \% \pm 6.0 \% \mathrm{BL}$ in females. Compared with rats that had been previously treated with vehicle, rats that had been previously treated with THC showed increased allodynia (i.e., decreased mechanical thresholds) on day 8 , and this effect did not differ between the sexes (THC, $\mathrm{F}_{4,145}=3.00, P=$ 0.020) (Supplemental Fig. 1). However, this THC effect was not significant at any single dose.

Figure 2, $\mathrm{C}$ and D shows that in the CBD experiment, CFA decreased mechanical threshold to $54.5 \% \pm 4.2 \% \mathrm{BL}$ in males and $51.7 \% \pm 4.2 \% \mathrm{BL}$ in females. Although its effects were small compared with THC, CBD was also antiallodynic compared with vehicle treatment $\left(\mathrm{CBD}, \mathrm{F}_{4,198}=2.51, P=\right.$ 0.043). CBD effects did not vary significantly between the sexes. Only $2.5 \mathrm{mg} / \mathrm{kg}$ produced a significant antiallodynic effect $(P=0.014$, Dunnett's), and this effect did not differ between acutely and repeatedly administered CBD (see Fig. 3 for CBD effects on von Frey pooled across sexes). On day 8, CFA + vehicle-treated rats had decreased mechanical thresholds compared with their baselines: $50.4 \% \pm 6.5 \% \mathrm{BL}$ in males and $45.2 \% \pm 4.9 \%$ BL in females. Mechanical thresholds in rats previously treated with CBD did not differ from those in rats previously treated with vehicle (Supplemental Fig. 1).

Heat Hyperalgesia. To gauge sex differences in THC and CBD effects on thermal nociception and determine whether tolerance develops to THC's or CBD's effects against thermal nociception, latency to respond to noxious heat was measured on day 4 and day 8 (see Supplemental Table 1 for planned comparisons in the THC experiment and Supplemental Table 2 for planned comparisons in the CBD experiment). Figure 4 shows the mean of day 4 time course data on the Hargreaves test in males and females treated acutely versus repeatedly with THC (Fig. 4, A and B) or CBD (Fig. 4, C and D). In the THC experiment, CFA decreased the response latency to noxious heat to $75.6 \% \pm 9.3 \% \mathrm{BL}$ in males and $62.4 \% \pm$ 9.7\% BL in females (see vehicle-treated groups). THC produced sex-dependent antihyperalgesia that differed between rats treated acutely versus repeatedly with THC ( $\operatorname{sex} \times$ THC $\times$ chronicity, $\mathrm{F}_{3,145}=2.69, P=0.049$ ). Subsequent analysis in only males showed that THC produced antihyperalgesia compared with vehicle treatment (THC, $\mathrm{F}_{4,71}=11.39, P<$ 0.001) (Fig. 4A), and this effect did not differ significantly between rats given THC acutely versus repeatedly. In contrast, in females, THC's antihyperalgesic effect was significantly reduced when THC was given repeatedly compared with acutely (THC $\times$ chronicity, $\mathrm{F}_{3,74}=5.48, P=0.002$ ), specifically at 2.0 and $4.0 \mathrm{mg} / \mathrm{kg}(P \leq 0.04$, Dunnett's $)$ (Fig. 4B). On day 8, the thermal threshold in CFA-treated rats was $101.1 \% \pm 14.7 \% \mathrm{BL}$ in males and $121.9 \% \pm 25.8 \% \mathrm{BL}$ in females (i.e., rats were no longer hyperalgesic to noxious heat). Thermal response latencies did not differ significantly between rats previously treated with THC and those previously treated with vehicle (Supplemental Fig. 2).

Figure 4, C and D shows that in the CBD experiment, CFAtreated control rats did not show a reduced thermal response latency on day $4(103.3 \% \pm 15.3 \% \mathrm{BL}$ in males and $98.3 \% \pm$ $12.8 \% \mathrm{BL}$ in females). In contrast to THC, CBD produced no 


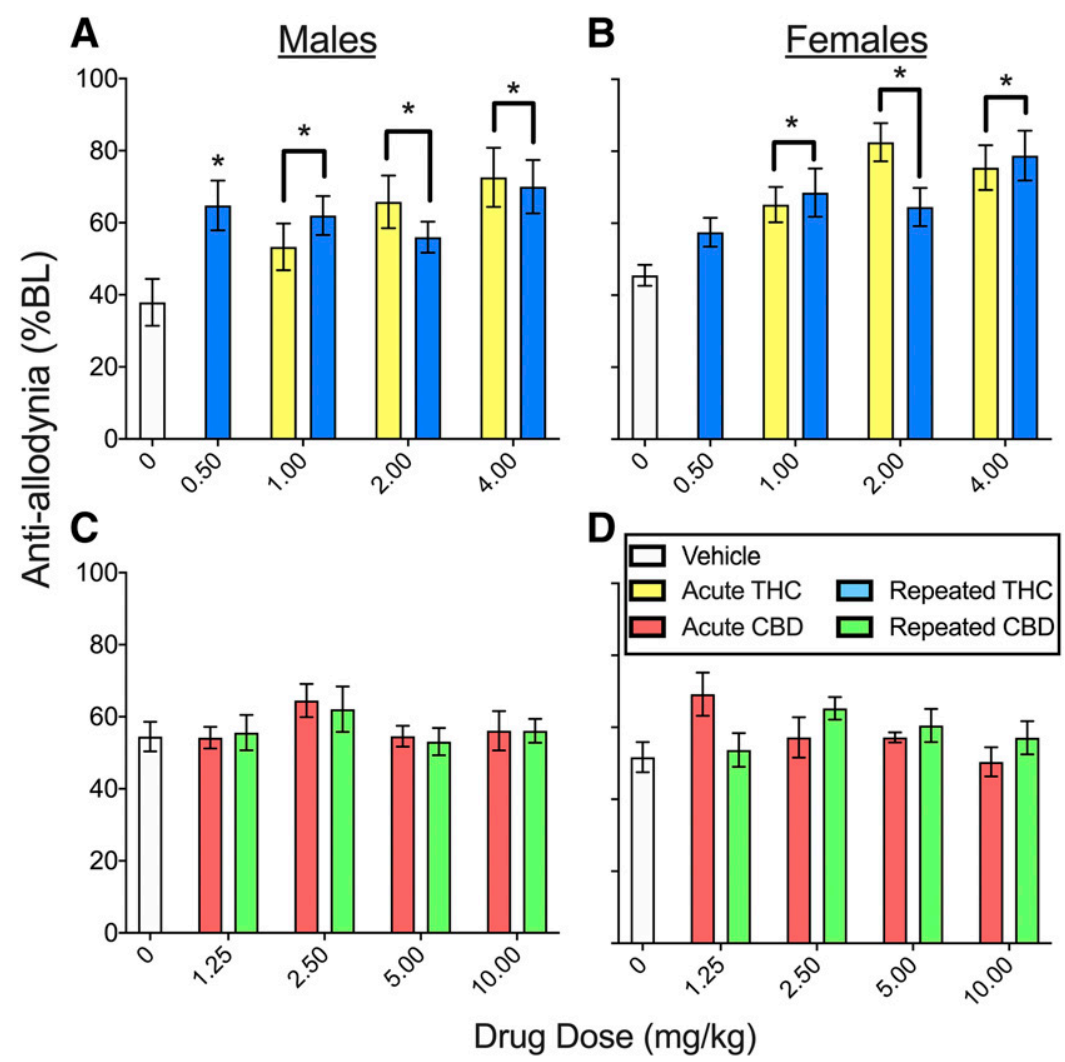

Fig. 2. THC and CBD effects in male and female rats on the von Frey test on day 4 (means of day 4 time course data are shown). In the THC experiment (A and $\mathrm{B}$ ), the mechanical threshold in rats that received vehicle treatment was $\sim 40 \% \mathrm{BL}$, indicative of allodynia. Acute and repeated THC were antiallodynic in both males (A) and females (B) (all $P \leq 0.010)$. In the $\mathrm{CBD}$ experiment $(\mathrm{C}$ and $\mathrm{D})$, the mechanical threshold in rats that received vehicle treatment was $\sim 55 \% \mathrm{BL}$, which was also indicative of allodynia. CBD $2.5 \mathrm{mg} / \mathrm{kg}$ was antiallodynic (significant effect not shown; see Supplemental Fig. 2 for data pooled across sexes). Each bar is the mean \pm 1 S.E.M. of $8-12$ male or female rats. *Significantly different from same-sex, vehicletreated controls $(P<0.05)$ significant effects on the Hargreaves test compared with vehicle treatment. On day 8 , the thermal response latency in CFA-treated control rats was $150.8 \% \pm 24.8 \%$ BL in males and $142.1 \% \pm 16.8 \% \mathrm{BL}$ in females. Thermal response latencies did not differ between rats previously treated with CBD and those previously treated with vehicle (Supplemental Fig. 2).

Weight-Bearing. In the CFA model, an efficacious analgesic would restore use of the inflamed hindpaw, and thus THC and CBD effects on hindpaw weight-bearing were measured on day 4 and day 8 (see Supplemental Table 1 for planned comparisons in the THC experiment and Supplemental Table 2 for planned comparisons in the CBD experiment). Figure 5 shows

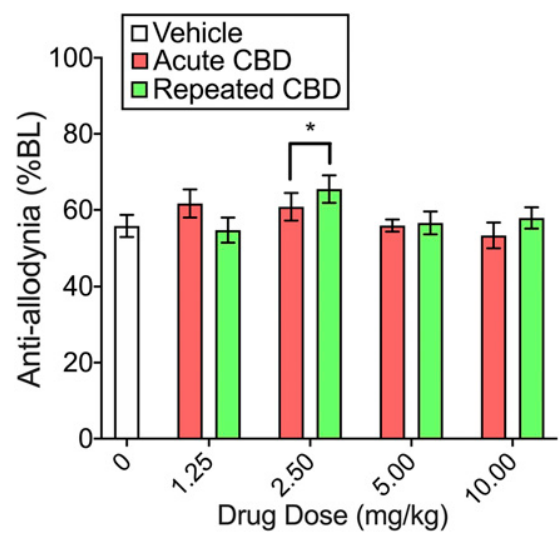

Fig. 3. CBD effects on the von Frey test on day 4, with data pooled across sexes (mean of day 4 time course data are shown). CBD $2.5 \mathrm{mg} / \mathrm{kg}$ was antiallodynic; this effect was not different when CBD was given acutely vs. repeatedly. Each bar is the mean \pm 1 S.E.M. of $16-24$ rats. *Significantly different from vehicle-treated controls $(P<0.05)$. the mean of day 4 time course data for weight-bearing on the inflamed paw for THC (Fig. 5, A and B) and CBD (Fig. 5, C and D). In the THC experiment, CFA decreased weight-bearing on the inflamed paw to $62.2 \% \pm 5.1 \% \mathrm{BL}$ in males and $55.5 \% \pm$ $4.2 \% \mathrm{BL}$ in females (see vehicle-treated groups). THC increased weight-bearing on the inflamed paw in both sexes (dose, $\mathrm{F}_{4,145}=2.74, P=0.031$ ). Although males had higher $\%$ BL than females (sex, $\mathrm{F}_{1,145}=4.17, P=0.043$ ), there were no sex differences in THC's effect on weight-bearing ( $\mathrm{sex} \times \mathrm{THC}$, $\mathrm{F}_{4,145}=0.60, P=0.66$ ). Analysis in only males showed that THC did not affect weight-bearing (Fig. 5A). However, in females THC increased weight-bearing on the inflamed paw (THC, $\mathrm{F}_{4,74}=3.44, P=0.012$ ), and this effect was greater in females treated repeatedly compared with acutely (chronicity, $\mathrm{F}_{1,74}=7.15, P=0.009$ ) (Fig. 5B). CFA-induced reductions in weight-bearing on the inflamed paw remained approximately the same on day 8 as they were on day 4 in vehicle-treated controls of both sexes $(\sim 60 \% \mathrm{BL})$. Compared with rats previously treated with vehicle, rats previously treated with THC placed more weight on the inflamed paw when tested on day 8 (THC, $\mathrm{F}_{4,145}=3.37, P=0.011$ ), with no significant difference between rats that had received THC acutely versus repeatedly (Supplemental Fig. 3).

In the CBD experiment (Fig. 5, C and D), CFA decreased weight-bearing on the inflamed paw to $66.2 \pm 5.2 \% \mathrm{BL}$ in males and $65.2 \% \pm 6.0 \% \mathrm{BL}$ in females (see vehicle-treated controls). CBD increased weight-bearing on the inflamed paw compared with vehicle treatment $\left(\mathrm{CBD}, \mathrm{F}_{4,198}=2.49, P=0.045\right)$, although this effect was not significant at any single dose. On day $8, \mathrm{CBD}$ treated rats did not differ from controls (Supplemental Fig. 3). 
A

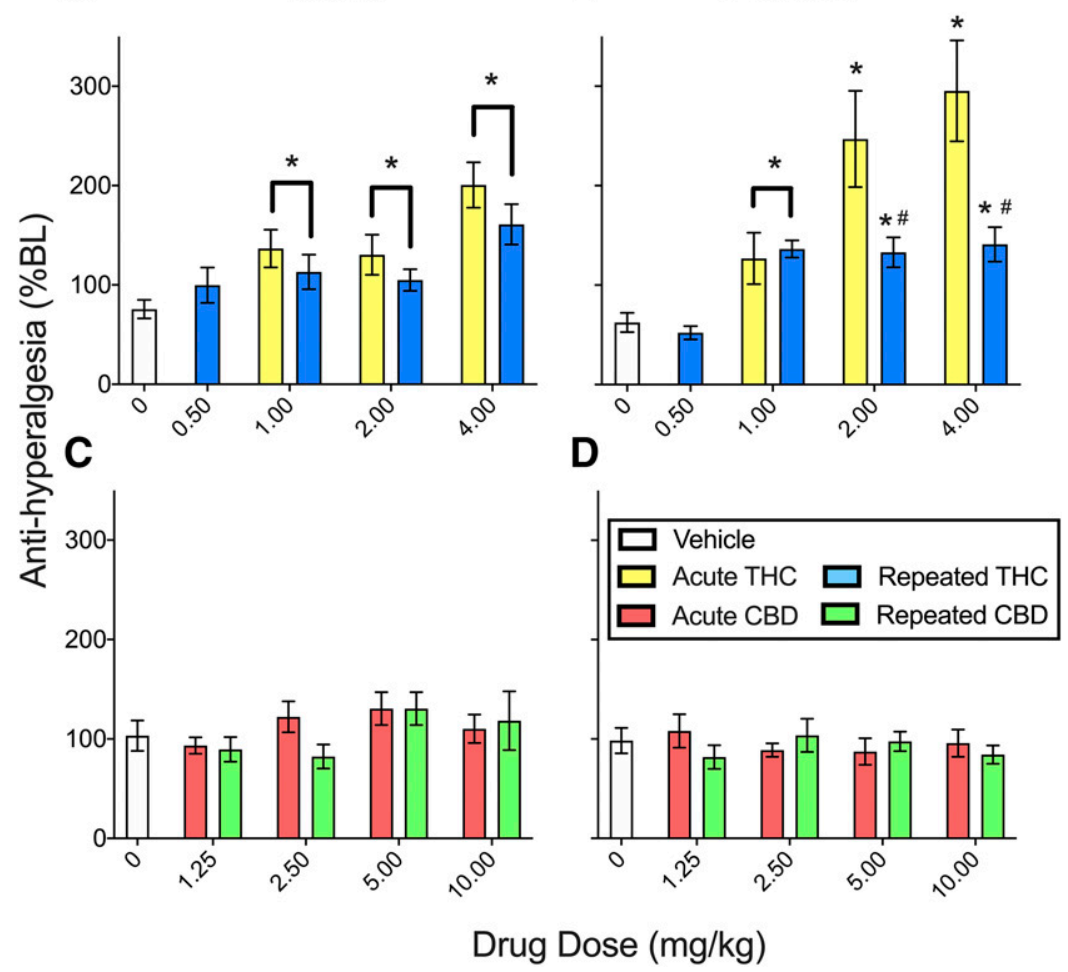

Fig. 4. THC and CBD effects in male and female rats on the Hargreaves test on day 4 (means of day 4 time course data are shown). In the THC experiment (A and B), the thermal response latency in rats that received vehicle was $\sim 75 \% \mathrm{BL}$, indicative of hyperalgesia. THC produced dosedependent antihyperalgesia that was greater in females than males. In males, all doses of THC were antihyperalgesic, and no tolerance developed when THC was given repeatedly. In females, THC $1-4 \mathrm{mg} / \mathrm{kg}$ was antihyperalgesic, and tolerance developed to this effect when THC was given repeatedly. In the CBD experiment (C and D), the thermal response latency in vehicle-treated rats was $\sim 100 \%$ BL, indicating no hyperalgesia. CBD did not alter thermal response latencies in either sex. Each bar is the mean \pm 1 S.E.M. of $8-12$ male or female rats. *Significantly different from same-sex, vehicle-treated controls $(P<0.05)$; \#Significantly different from same dose administered acutely $(P<0.05)$
Hindpaw Edema. To determine THC and CBD effects on edema, hindpaw thickness was measured on day 4 and day 8 (see Supplemental Table 1 for planned comparisons in the THC experiment and Supplemental Table 2 for planned comparisons in the CBD experiment). Figure 6 shows THC (Fig. 6, A and B) and CBD (Fig. 6, C and D) effects on paw thickness (which was measured at the 240-minute time point only) on day 4 . In the THC experiment, CFA increased paw

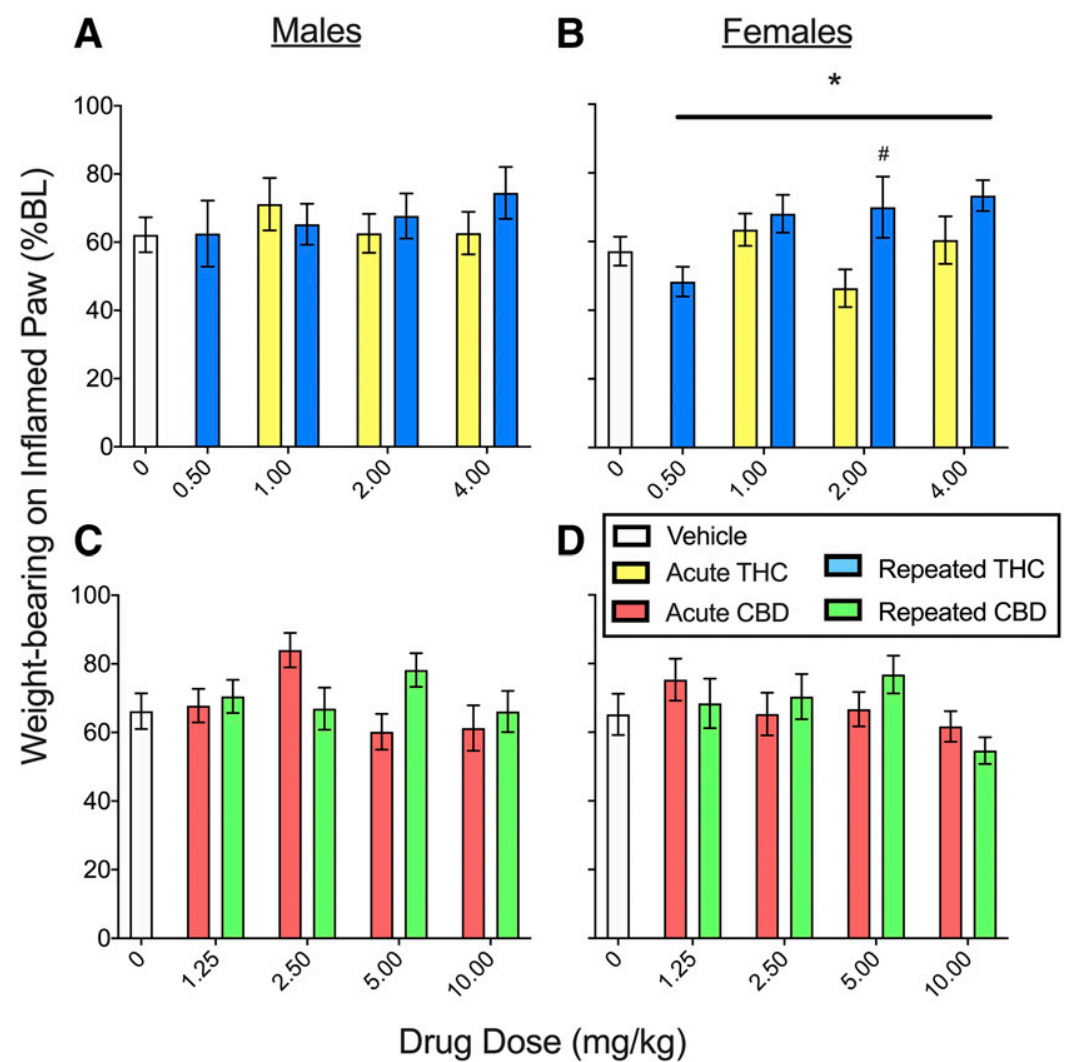

Fig. 5. THC and CBD effects in male and female rats on the incapacitance (weight-bearing) test on day 4 (means of day 4 time course data are shown). In the THC experiment (A and $\mathrm{B}$ ), weight-bearing on the inflamed paw was $\sim 60 \% \mathrm{BL}$ in rats treated with vehicle, indicating paininduced suppression of weight-bearing. THC produced small increases in weight-bearing on the inflamed paw in females (B) but not males (A); increases in weight-bearing in females were larger when THC was given repeatedly compared with acutely (B). In the CBD experiment $(\mathrm{C}$ and $\mathrm{D})$, weight-bearing on the inflamed paw was $\sim 65 \% \mathrm{BL}$ in rats treated with vehicle, indicating pain-induced suppression of weight-bearing. CBD produced a small increase in weight-bearing on the inflamed paw that was similar in both sexes $(C$ and $D)$. Each bar is the mean \pm 1 S.E.M. of 8-12 male or female rats. *Significantly different from same-sex, vehicle-treated controls $(P<0.05)$; \#significantly different from same dose administered acutely $(P<0.05)$. 
thickness to $205.3 \% \pm 7.0 \% \mathrm{BL}$ in males and $207.8 \% \pm$ $5.7 \% \mathrm{BL}$ in females (see vehicle-treated groups). Although females had larger \% BL paw thickness than males on day 4 (sex, $\left.\mathrm{F}_{1,145}=4.85, P=0.029\right)$. THC did not reduce paw thickness in either sex $\left(\mathrm{THC}, \mathrm{F}_{4,145}=1.41, P=0.235\right)$. On day 8, CFA + vehicle-treated control rats had increased paw thickness compared with their baselines: $161.1 \% \pm 5.1 \%$ BL in males and $175.6 \% \pm 6.1 \% \mathrm{BL}$ in females. Paw thickness did not differ significantly between rats that were previously treated with THC compared with those previously treated with vehicle (Supplemental Fig. 4).

In the CBD experiment (Fig. 6, C and D), CFA increased paw thickness to $219.0 \% \pm 5.7 \% \mathrm{BL}$ in males and $218.9 \% \pm$ $5.8 \% \mathrm{BL}$ in females (see vehicle-treated controls). Although females had larger \% BL paw thickness than males on day 4 (sex, $\left.\mathrm{F}_{1,145}=7.28, P=0.008\right)$, there was no significant sex difference in CBD's effect ( $\operatorname{sex} \times \mathrm{CBD}, \mathrm{F}_{4,145}=1.44, P=0.223$ ). $\mathrm{CBD}$ decreased paw thickness compared with vehicle $\left(\mathrm{CBD}, \mathrm{F}_{4,198}=2.72, P=\right.$ 0.031 ); post hoc analysis showed that only the highest dose of CBD (10 mg/kg) significantly decreased paw thickness compared with vehicle ( $P=0.011$, Dunnett's) (see Supplemental Fig. 5 for CBD effects on paw thickness pooled across sexes).

On day 8, CFA-treated rats had increased paw thickness compared with baseline: $171.6 \% \pm 3.9 \% \mathrm{BL}$ in males and $186.8 \% \pm 5.4 \% \mathrm{BL}$ in females. Although females had larger $\%$ BL paw thickness than males on day $8\left(\mathrm{sex}, \mathrm{F}_{1,145}=20.49\right.$, $P<0.001)$ and CBD decreased paw thickness $\left(\mathrm{CBD}, \mathrm{F}_{4,145}=\right.$ $2.72, P=0.031$ ), there was no sex difference in CBD's effect ( $\operatorname{sex} \times \mathrm{CBD}, \mathrm{F}_{4,145}=1.28, P=0.280$ ). Subsequent analysis in only males revealed that previous CBD treatment did not significantly decrease paw thickness compared with vehicle treatment. However, analysis in only females showed that rats previously treated with CBD had decreased paw thickness on day 8 compared with females previously treated with vehicle $\left(\mathrm{CBD}, \mathrm{F}_{4,97}=3.22, P=0.016\right)$; post hoc analysis showed that only females previously treated with the highest dose of CBD (10 $\mathrm{mg} / \mathrm{kg})$ had decreased paw thickness on day 8 compared with females treated previously with vehicle $(P=$ 0.013, Dunnett's) (Supplemental Fig. 4).

Locomotor Activity. To measure both pain-suppressed behavior and drug-induced sedation, locomotor activity was measured on day 4 and day 8 (see Supplemental Table 1 for planned comparisons in the THC experiment and Supplemental Table 2 for planned comparisons in the CBD experiment). Figure 7 shows the mean of day 4 time course data on the locomotor activity test for THC (Fig. 7, A and B) and CBD (Fig. 7, $\mathrm{C}$ and D). In the THC experiment, CFA decreased locomotor activity to $42.4 \% \pm 3.8 \% \mathrm{BL}$ in males and $50.5 \% \pm 7.2 \% \mathrm{BL}$ in females (see vehicle-treated controls). Compared with vehicletreated controls, THC reduced locomotor activity, and this effect did not differ significantly between the sexes $\left(\mathrm{THC}, \mathrm{F}_{4,145}=\right.$ $14.38, P<0.001$; THC $\left.\times \operatorname{sex}, \mathrm{F}_{4,145}=0.60, P=0.664\right)$. All THC doses except $0.5 \mathrm{mg} / \mathrm{kg}$ decreased locomotor activity $(P \leq 0.007$, Dunnett's). THC-induced locomotor suppression did not differ between rats treated acutely versus repeatedly with THC $\left(\mathrm{THC} \times\right.$ chronicity, $\left.\mathrm{F}_{3,145}=1.33, P=0.269\right)$ (see Supplemental Fig. 6 for THC effect on locomotor activity pooled across sexes).

Locomotor activity was decreased on day 8 compared with baseline, to $62.9 \% \pm 6.0 \% \mathrm{BL}$ in control males and $64.7 \% \pm$ $6.3 \% \mathrm{BL}$ in control females. Surprisingly, rats that were
Males

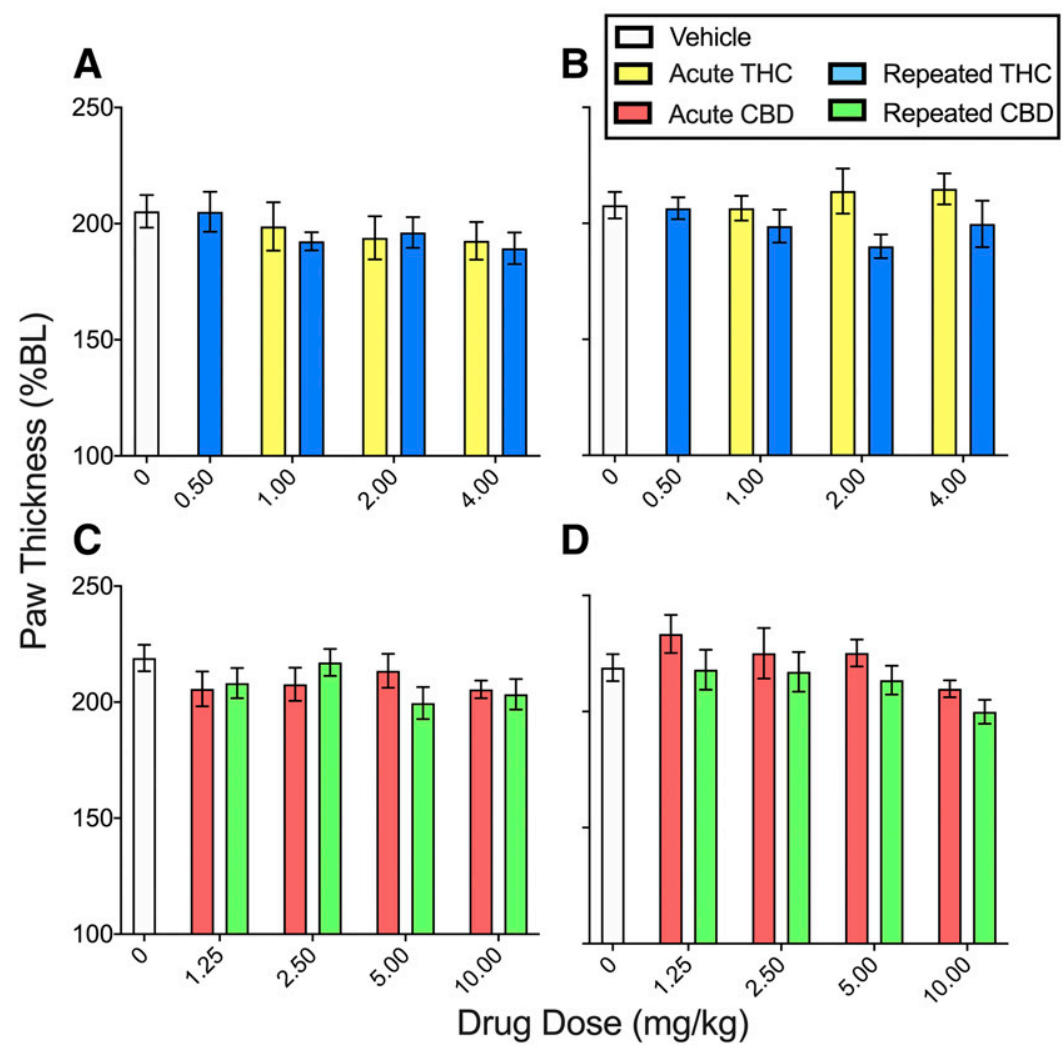

Fig. 6. THC and CBD effects in male and female rats on paw thickness (edema) on day 4 (measured 240 minutes postinjection). In the THC experiment (A and $\mathrm{B}$ ), paw thickness was $\sim 205 \% \mathrm{BL}$ in rats treated with vehicle, indicating edema. THC did not alter paw thickness in either sex. In the CBD experiment (C and $\mathrm{D}$ ), paw thickness was $\sim 220 \% \mathrm{BL}$ in rats treated with vehicle, indicating edema. CBD decreased paw thickness at the highest dose tested, $10 \mathrm{mg} / \mathrm{kg}$, similarly in both sexes (significant effect not shown; see Supplemental Fig. 6 for data pooled across sexes). Each bar is the mean \pm 1 S.E.M. of $8-12$ male or female rats. 
A

B

Females

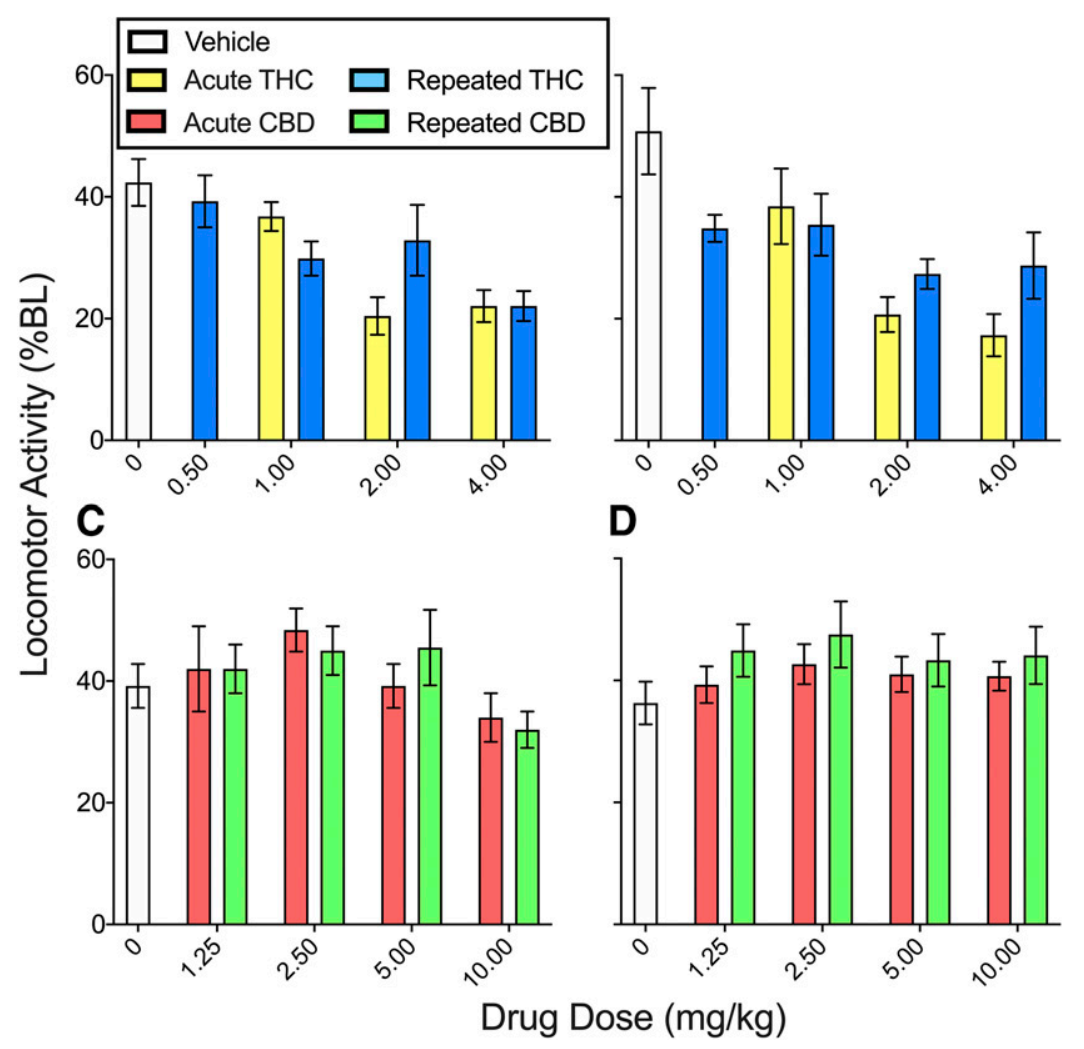

Fig. 7. THC and CBD effects in male and female rats on the locomotor activity test on day 4 (means of day 4 time course data are shown). In the THC experiment (A and B), locomotor activity in rats that received vehicle was $\sim 45 \% \mathrm{BL}$, indicating pain-suppressed behavior. THC decreased locomotor activity similarly in both sexes (significance not shown; see Supplemental Fig. 7 for data pooled across sexes). In the CBD experiment (C and D), locomotor activity in rats that received vehicle was $\sim 40 \% \mathrm{BL}$, indicating pain-suppressed behavior. CBD $2.5 \mathrm{mg} / \mathrm{kg}$ increased locomotor activity similarly in both sexes (significance not shown; see Supplemental Fig. 7 for data pooled across sexes). Each bar is the mean \pm 1 S.E.M. of 8-12 male or female rats.

previously treated with THC showed decreased locomotor activity on day 8 compared with rats that were treated with vehicle (THC, $\mathrm{F}_{4,145}=2.81, P=0.028$ ) (Supplemental Fig. 7).

In the CBD experiment (Fig. 7, C and D), CFA decreased locomotor activity to $39.2 \% \pm 3.6 \% \mathrm{BL}$ in males and $36.3 \% \pm$ $3.5 \% \mathrm{BL}$ in females (see vehicle-treated controls). CBD increased locomotor activity compared with vehicle treatment $\left(\mathrm{CBD}, \mathrm{F}_{4,198}=2.64, P=0.035\right)$, and this effect did not differ between the sexes or between rats treated acutely versus repeatedly. Post hoc analysis showed that CBD $2.5 \mathrm{mg} / \mathrm{kg}$ significantly increased locomotor activity $(P=$ 0.026 , Dunnett's). On day 8, CFA + vehicle-treated control rats showed decreased locomotor activity compared with their baselines: $52.9 \% \pm 6.6 \%$ BL in males and $60.8 \% \pm$ $7.7 \% \mathrm{BL}$ in females. On day 8 , rats that were previously treated with CBD on days 1-4 did not locomote differently than rats that were previously treated with vehicle (Supplemental Fig. 7).

Body Weight. To gauge rats' overall health, body weight was measured on days 1-4 and day 8 . Figure 8 shows body weight in males and females treated repeatedly with THC or CBD. By day 2, CFA + vehicle-treated rats' body weight had dropped to about $95 \%$ of baseline (day 1) body weight in both THC and CBD experiments. Despite the fact that THC reduced some pain-related behaviors, rats repeatedly treated with THC lost more weight than rats repeatedly treated with vehicle (day $\times$ THC, $\left.\mathrm{F}_{7,136}=8.43, P<0.001\right)($ Fig. $8, \mathrm{~A}$ and $\mathrm{B})$. Further analysis on each day showed that rats treated with THC weighed significantly less than rats treated with vehicle on days $2,3,4$, and 8 (all $P<0.05$, Dunnett's).
On day 8, CFA + vehicle-treated (control) males had reduced \% BL body weight, whereas control females did not (day 8 , sex, $\mathrm{F}_{1,81}=4.81, P=0.031$ ). On day 8 , males that had received THC repeatedly weighed less than males that had received vehicle (THC, $\mathrm{F}_{4,40}=17.39, P<0.001$ ) (Fig. 8A). Interestingly, males that had been injected once with THC on day 4 also weighed less on day 8 than males that received vehicle (THC, $\mathrm{F}_{3,64}=3.07, P=0.034$ ); however, this effect was only significant at $2.0 \mathrm{mg} / \mathrm{kg}(P=0.027)$ (Supplemental Table 1). On day 8 , females that had received THC repeatedly on days 1-4 weighed less than female rats that had received vehicle (THC, $\mathrm{F}_{4,41}=3.89, P=0.009$ ) (Fig. $8 \mathrm{~B}$ ).

In contrast to THC, CBD did not reduce body weight. In fact, on day 8 , rats previously treated with CBD weighed significantly more than the vehicle group $\left(\mathrm{CBD}, \mathrm{F}_{4,98}=2.94, P=\right.$ 0.024) (Fig. 8, C and D). Post hoc analysis showed that rats treated repeatedly with 5.0 or $10.0 \mathrm{mg} / \mathrm{kg}$ CBD were more likely than vehicle-treated rats to have returned to their day 1 body weights $(P<0.05)$. On day 8 , even rats that had been injected once with $\mathrm{CBD}$ on day 4 weighed more than rats that had received vehicle $\left(\mathrm{CBD}, \mathrm{F}_{4,100}=3.86, P=0.006\right)$. Post hoc analysis showed that a single administration of CBD $10 \mathrm{mg} / \mathrm{kg}$ on day 4 restored body weight to day 1 levels by day 8 in both sexes $(P=0.002)$.

\section{Experiment 2: Acute Versus Repeated THC or CBD Effects on Serum Cytokines and Spleen Weight}

In experiment 2 we determined how acute and repeated THC or CBD exposure affected serum cytokine levels in our model of CFA-induced inflammation. Figure 9 shows THC and 


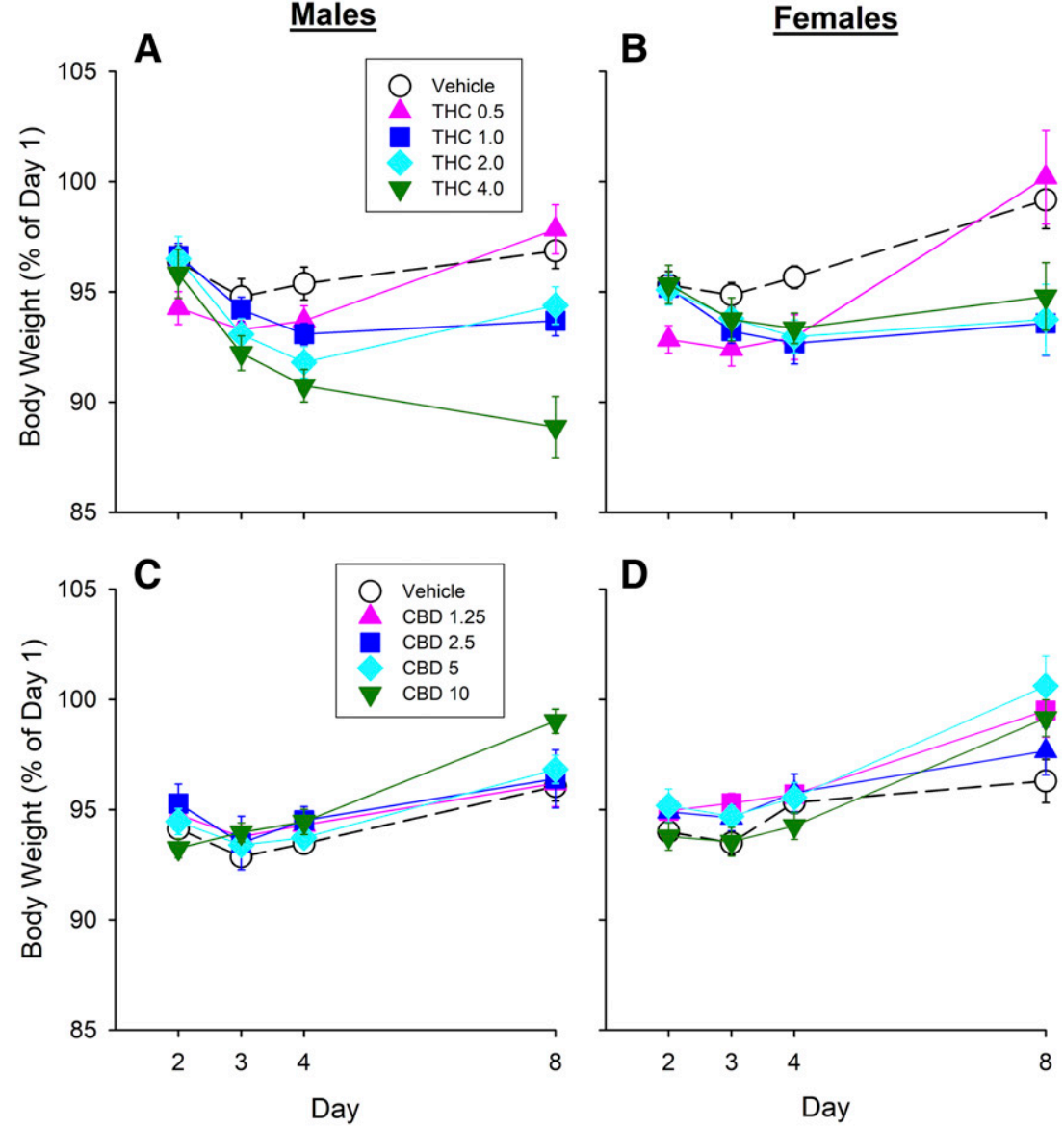

Fig. 8. Body weight during the course of Experiment 1 in male $(\mathrm{A}$ and $\mathrm{C})$ and female $(\mathrm{B}$ and $\mathrm{D})$ rats treated repeatedly with THC (A and $\mathrm{B})$ or $\mathrm{CBD}(\mathrm{C}$ and $\mathrm{D})$. CFA decreased body weight in vehicle-treated rats of both sexes to $\sim 95 \%$ BL. Male and female rats treated with THC repeatedly weighed significantly less than rats treated with vehicle, whereas CBD treatment restored day 1 body weight in both sexes. Each point is the mean \pm 1 S.E.M. of $8-12$ male or female rats.
CBD effects on serum cytokine concentrations in male and female rats with or without hindpaw inflammation measured on day 4 (3 days after intraplantar injection of mineral oil or CFA). There were large sex differences in some cytokines: females had higher levels of TNF- $\alpha$, IL- $1 \beta$, and IL- 6 than males (Fig. 9, A-F; TNF- $\alpha$ : $\mathrm{F}_{1,84}=34.21, P<0.001$; IL-1 $\beta$ : $\left.\mathrm{F}_{1,84}=16.26, P<0.001 ; \mathrm{IL}-6: \mathrm{F}_{1,84}=71.83, P<0.001\right)$, whereas no sex differences were observed in serum IL-10 or IFN- $\gamma$. Analysis in only males showed that CFA injection into the hindpaw significantly increased TNF- $\alpha$, IL- $1 \beta$, and IL-6 concentrations (TNF- $\alpha: \mathrm{F}_{1,42}=32.09, P<0.001 ;$ IL- $1 \beta$ : $\mathrm{F}_{1,42}=10.63, P=0.002$; IL-6: $\left.\mathrm{F}_{1,42}=14.53, P<0.001\right)$. In contrast, CFA did not significantly affect these cytokines in females.

Although TNF- $\alpha$, IL- 6 , and IFN- $\gamma$ concentrations were not altered by either acutely or repeatedly administered THC, THC increased IL- $1 \beta$ levels (Fig. 9, C and D; THC, $\mathrm{F}_{2,84}=3.79$, $P=0.027$ ). Although there were sex differences in IL- $1 \beta$ (sex, $\mathrm{F}_{1,84}=16.26, P<0.001$ ), there were no sex differences in THC's effect on IL-1 $\beta$ (THC $\times$ sex, $\left.\mathrm{F}_{2,84}=0.15, P=0.859\right)$. Subsequent analysis in only males showed that THC increased IL-1 $\beta$ (Fig. 9C; THC, $\mathrm{F}_{1,42}=3.26, P=0.048$ ). Post hoc analysis showed that only repeated administration of THC increased serum IL-1 $\beta$ in males ( $P=0.042$, Dunnett's); although this effect was not dependent on pain state $(\mathrm{THC} \times$ CFA, $\mathrm{F}_{2,42}=1.85, P=0.169$ ). Follow-up analysis in only mineral oil-treated males and only CFA-treated males showed that THC did not alter IL- $1 \beta$ in either pain condition. THC also did not alter IL- $\beta$ in females.
THC's effects on IL-10 were both sex- and inflammatory state-dependent (Fig. 9, G and H; sex $\times \mathrm{THC} \times \mathrm{CFA}_{2,84}=$ 4.04, $P=0.021$ ). Subsequent analysis in control (mineral oil-treated) rats showed that THC's effects were sexdependent ( $\left.\operatorname{sex} \times \mathrm{THC}, \mathrm{F}_{2,42}=3.62, P=0.035\right)$; however, follow-up analysis within each sex showed no significant effect of THC on serum IL-10 concentration in control rats of either sex. Additionally, THC did not alter serum IL-10 in male or female rats with inflammation.

In contrast to THC, CBD reduced IL-10 and IFN- $\gamma$ (IL-10: $\left.\mathrm{F}_{2,84}=7.17, P=0.001 ; \mathrm{IFN}-\gamma: \mathrm{F}_{2,84}=85.72, P<0.001\right)$. Analysis in only healthy (mineral oil-treated) rats showed that CBD decreased IL-10, and this effect differed between the sexes $\left(\mathrm{CBD} \times \operatorname{sex}, \mathrm{F}_{2,42}=4.40, P=0.043\right)$. Subsequent analysis in only healthy (mineral oil-treated) males showed that an acute dose of CBD decreased IL-10 (Fig. 9G; $P=0.021$, Dunnett's), but repeated dosing did not. In healthy females, both acute and repeated CBD treatment decreased IL-10 (Fig. $9 \mathrm{H}$; acute: $P=0.001$; repeated: $P=0.002$, Dunnett's). Additionally, both acute and repeated CBD decreased IFN- $\gamma$ in both healthy (mineral oil-treated) and CFA-treated males and females (Fig. 9, I and J; $P<0.010$, Dunnett's).

CBD decreased TNF- $\alpha$ in CFA-treated males but not in healthy males (Fig. 9A; CBD $\left.\times \mathrm{CFA}, \mathrm{F}_{2,34}=3.52, P=0.041\right)$ or in females (control and CFA-treated) (Fig. 9B). CBD also decreased IL- $1 \beta$ levels in males and females (Fig. 9C, males: CBD, $\mathrm{F}_{1,30}=6.05, P=0.006$; Fig. 9D, females: CBD, $\mathrm{F}_{2,37}=$ $9.61, P<0.001)$ when given acutely or repeatedly $(P \leq 0.020)$. Further analysis showed that CBD decreased IL- $1 \beta$ in healthy 


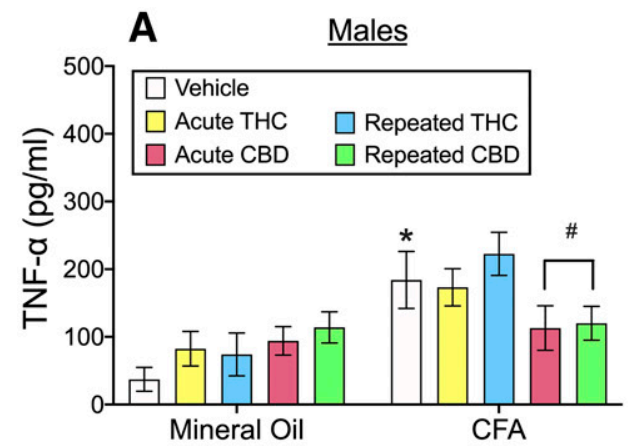

B Females
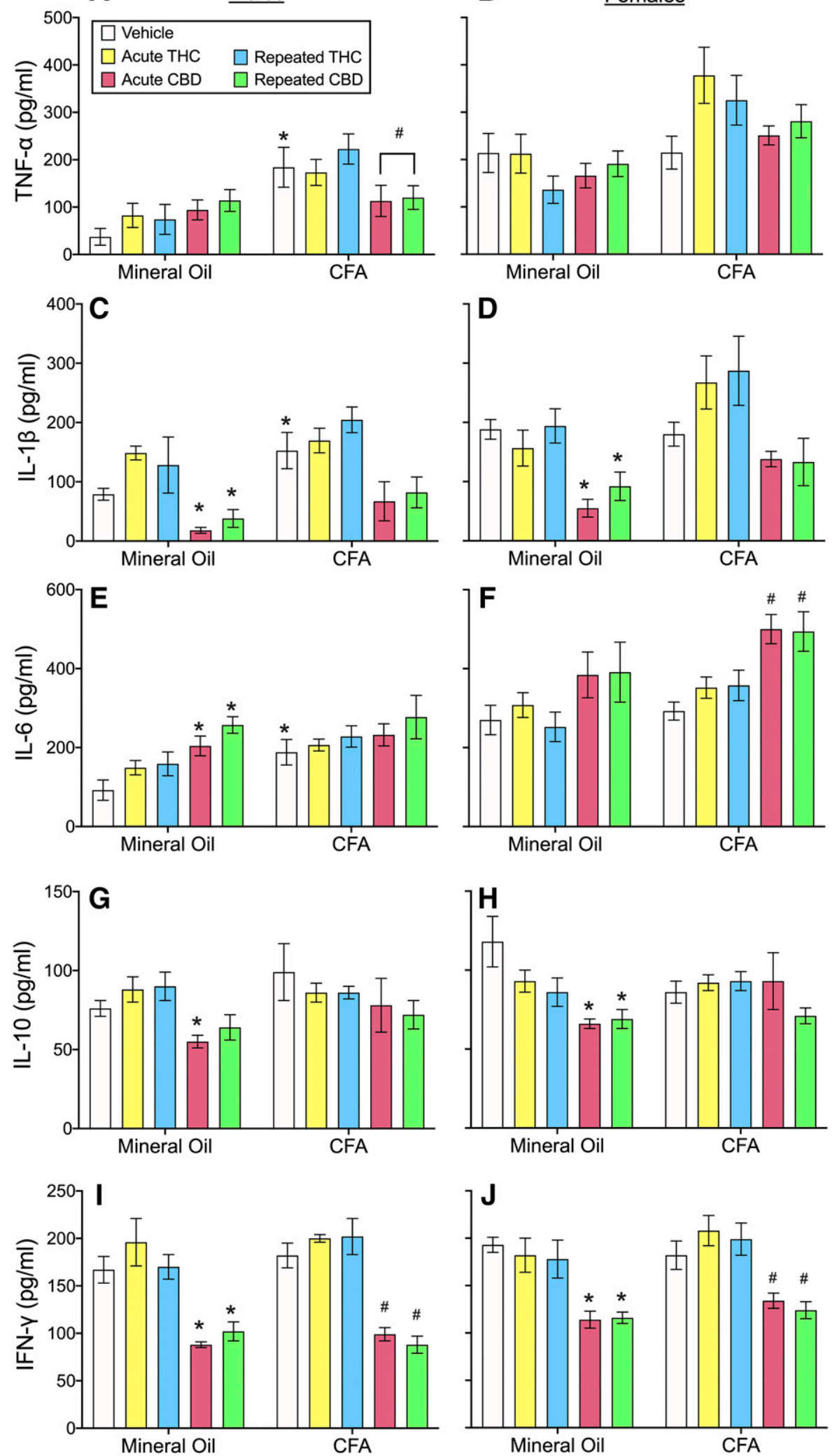

Fig. 9. Experiment 2: serum cytokine concentrations in male (left panels: A, C, D, F, G, and I) and female (right panels: B, D, F, H, and J) rats, at 240 minutes postinjection on day 4 . (A and $\mathrm{B}$ ) CFA increased TNF- $\alpha$ in males (A) but not females (B); THC did not alter TNF- $\alpha$, but CBD decreased TNF- $\alpha$ in only healthy males. (C and D) CFA increased IL- $1 \beta$ in males (C) but not females (D); repeated THC increased IL-1 $\beta$ in males, but subsequent analysis showed this effect was not significant in either mineral oilor CFA-treated rats. THC did not alter IL- $1 \beta$ in females. CBD decreased IL- $1 \beta$ similarly in healthy males and females. ( $\mathrm{E}$ and F) CFA increased IL-6 in males (E) but not females (F); THC did not alter IL-6, and CBD increased IL-6 only in healthy males and CFA-treated females. ( $\mathrm{G}$ and $\mathrm{H}$ ) CFA did not affect serum IL-10; THC effects on IL-10 were sex- and pain statedependent, and CBD decreased IL-10 similarly in both sexes, and post hoc analysis showed this was significant only in mineral oil-treated rats. (I and F) CFA did not affect serum IFN- $\gamma$; THC did not alter serum IFN- $\gamma$, whereas CBD reduced IFN- $\gamma$ in both sexes. Each bar is the mean \pm 1 S.E.M. of four to eight male or female rats. *Significantly different than same-sex, mineral oil + vehicle-treated control group $(P<0.05)$; " significantly different than same-sex, CFA + vehicle-treated group $(P<0.05)$. (mineral oil-treated) males and females (males: CBD, $\mathrm{F}_{2,14}=$ $7.85, P=0.005$; females, $\mathrm{CBD} \mathrm{F}_{2,18}=15.44, P<0.001$ ) but not in CFA-treated males and females. CBD also significantly increased IL- 6 in both sexes (Fig. 9, E and F; CBD, $\mathrm{F}_{2,83}=$
15.44, $P<0.001$ ). Although there were sex differences in IL-6 serum concentration ( $\mathrm{sex}, \mathrm{F}_{1,83}=63.23, P<0.001$ ), there were no sex differences in CBD-induced changes in IL-6 (sex $\times$ $\left.\mathrm{CBD}, \mathrm{F}_{1,83}=1.14, P=0.325\right)$. CBD increased IL-6 in healthy 
males (Fig. 9E; CBD, $\mathrm{F}_{2,41}=9.19, P=0.001$ ) but not in CFAtreated males. Post hoc analysis in healthy males showed that both acute and repeated administration of CBD increased IL-6 (acute, $P=0.021$; repeated, $P<0.001)$. In contrast, in healthy females CBD did not alter IL-6, but in CFA-treated females both acute and repeated administration of CBD increased IL-6 (Fig. 9F; $P<0.002$, Dunnett's).

As a percent of body weight, spleen weight was greater in females than males (sex, $\mathrm{F}_{1,84}=73.46, P<0.001$ ) (Supplemental Fig. 8). Subsequent analysis in males showed that CFA increased spleen weight $\left(\mathrm{F}_{1,42}=10.13, P=0.003\right)$, but neither THC nor CBD altered spleen weight. In females, CFA did not increase spleen weight, and neither THC nor CBD altered spleen weight.

\section{Discussion}

In the current study, THC produced dose-dependent antiallodynia and antihyperalgesia, increased weight-bearing on the inflamed paw, and decreased locomotion at doses that did not reduce edema. THC effects on pain-related behaviors are consistent with previous studies, in which intraperitoneal THC alleviated hyperalgesia in male rats with arthritis (Cox and Welch, 2004) and carrageenan-induced hindpaw inflammation (Rock et al., 2018) and was antiallodynic and antihyperalgesic in male and female rats with CFA-induced hindpaw inflammation (Craft et al., 2013). Acute THC was more potent/efficacious in females than males on the Hargreaves test (present study; Craft et al., 2013), but there were no sex differences in THC effect on other behavioral assays. Overall, the THC results agree with previous studies of THC's effects in rodent inflammatory pain models.

The present results indicate that THC maintains its antinociceptive efficacy over several days. Tolerance development was only observed on the Hargreaves test: large, dosedependent increases in latency to respond to noxious heat were evident in females treated acutely but not repeatedly with THC. Although THC-induced increases in nociceptive thresholds well above baseline were lost with repeated THC treatment, restoration of normal heat sensitivity (i.e., antihyperalgesia) was maintained. Thus, tolerance development observed on the Hargreaves test may not indicate a drawback therapeutically. Robust tolerance to THC's antinociceptive effect against noxious heat has been observed previously using acute heat-pain tests in healthy rodents (Bass and Martin, 2000; McKinney et al., 2008; Wakley et al., 2014; Greene et al., 2018). Somewhat surprising was the fact that rats in the present study did not become tolerant to THC's locomotor suppressant effect. Healthy male and female rats given twice-daily THC ( $\sim-10 \mathrm{mg} / \mathrm{kg}$ for 5-9.5 days) developed tolerance to THC-induced sedation in previous studies (Wiley et al., 2007; Wakley et al., 2014; Greene et al., 2018). Lack of tolerance to THC's hypolocomotor effect may be due to the relatively low doses and/or short treatment period used in the present study.

Failure of THC to reduce edema in the current study contrasts with previous studies demonstrating that oral THC decreased influenza-induced lung inflammation in female mice (Buchweitz et al., 2007), and intraperitoneal THC pretreatment decreased gut tissue inflammation in male rats with colitis (Jamontt et al., 2010) as well as carrageenaninduced hindpaw edema in male rats (Rock et al., 2018).
Differences in adjuvant type and timing of THC administration relative to induction of inflammation between previous studies and the present may explain differences in results. Additionally, we previously reported that intraperitoneal THC decreased paw thickness in male rats with CFA-induced paw edema, but THC was considerably more efficacious when administered intraplantar compared with intraperitoneally (Craft et al., 2013), suggesting that THC applied directly to the site of tissue inflammation is more effective than systemically administered THC at reducing edema.

In the current study, $2.5 \mathrm{mg} / \mathrm{kg}$ CBD was modestly antiallodynic and partially restored biased weight-bearing and pain-suppressed locomotion with no differences between males and females. Additionally, $10 \mathrm{mg} / \mathrm{kg}$ CBD reduced paw edema by approximately $15 \%$. In contrast, Costa et al. (2004) found that 4 days of once-daily oral CBD $(5 \mathrm{mg} / \mathrm{kg}$ ) starting 2 hours postcarrageenan reduced hindpaw edema by $40 \%$ in male rats. A single intraperitoneal injection of $5 \mathrm{mg} / \mathrm{kg}$ CBD also reduced zymosan A-induced hindpaw edema by $50 \%$ when given immediately after zymosan $\mathrm{A}$ and was antiallodynic in female mice 6 hours after zymosan A administration (Gallily et al., 2015). It is unclear to what extent route of administration, timing of CBD administration relative to induction of inflammation, adjuvant type, or other methodological factors explain the minimal CBD effects we observed compared with earlier studies.

CBD produced no effects on the Hargreaves test in the present study despite previous studies that consistently show antihyperalgesic effects of CBD against noxious heat (Costa et al., 2004; Hammell et al., 2016; Rock et al., 2018). Because CFA did not produce hyperalgesia in our CBD experiment (day 4 vehicle-treated rats responded at $\sim 101 \%$ of their preCFA baseline), antihyperalgesic effects of CBD could not be measured. It can be argued that the lack of CBD effect on the Hargreaves test simply reflects a lack of effect against noxious heat in rats with normal heat-pain thresholds. In this regard, the current results are consistent with previous studies showing that $\mathrm{CBD}$ has no effect on responses to acute heatpain in healthy rodents (Sofia et al., 1975; Sanders et al., 1979; Varvel et al., 2006; Booker et al., 2009; Britch et al., 2017).

The current study confirms some previous immunologic findings as well as revealing several novel findings. CFA increased serum TNF- $\alpha$, IL- $1 \beta$, and IL- 6 concentrations and spleen weight in males but not females in the present study, which is consistent with previous findings. The application of LPS to human peripheral blood mononuclear cells increased TNF- $\alpha$ release more in cells from males than females (Moxley et al., 2002), injected LPS increased plasma IL- $1 \beta$ more in isolated male than female rats (Yee and Prendergast, 2010), and LPS increased TNF- $\alpha$ and IL- 6 concentrations more in blood samples from men than women (Lefevre et al., 2012). Given the role of TNF- $\alpha$, IL- $1 \beta$, and IL- 6 in autoimmune diseases (Moudgil and Choubey, 2011) and higher rates of several autoimmune diseases in women than men (Gleicher and Barad, 2007; Zandman-Goddard et al., 2007), further studies investigating sex differences in cytokine responses to pathogens/inflammatory agents should be conducted to document the changes in cytokines during the transition from protective immunity to autoimmunity. Sex difference studies will provide crucial groundwork for comparing cannabinoid effects on immune function in males and females. 
Two studies have demonstrated that THC or synthetic cannabinoids can modulate cytokines: THC application to rat microglial cells decreased TNF- $\alpha$, IL- $1 \beta$, and IL- 6 mRNA 6 hours post-LPS (Puffenbarger et al., 2000), and WIN55,2122 and HU-210 decreased serum TNF- $\alpha$, IL-6, and IFN- $\gamma$ but increased IL-10 in endotoxic male mice at 1.5-3 hours postLPS challenge (Smith et al., 2000). In the present study, THC did not alter TNF- $\alpha$, IL-6, IL-10, or IFN- $\gamma$ and increased IL-1 $\beta$ in male rats. It is possible that THC's immune effects peak during early stages of the innate response (within 24 hours postadjuvant); if so, peak effects were missed by measuring cytokines 3 days post-CFA. Additionally, THC's effects may be adjuvant-dependent.

Several studies have observed CBD effects on cytokines. CBD decreased IFN- $\gamma$ in the draining lymph node and TNF- $\alpha$ release from synovial cells in arthritic male mice and blocked LPS-induced increases in serum TNF- $\alpha$ in female mice (Malfait et al., 2000). CBD given immediately after zymosan A-induced hindpaw inflammation decreased serum TNF- $\alpha$ in female mice (Gallily et al., 2015, 2018). We found that CBD decreased TNF- $\alpha$ in male rats and IFN- $\gamma$ in both sexes, consistent with Malfait et al. (2000). CBD did not decrease TNF- $\alpha$ in females in our study in contrast to the studies by Gallily and colleagues. More studies are needed to confirm whether the sex differences in cannabinoid effects we observed are reliable, whether they are species-specific, whether they generalize to other adjuvants/disease states, and whether they reflect different time courses or potencies of CBD effect between males and females. Although inhibition of inflammatory cytokines during pathologic pain states can be beneficial, immune suppression in individuals not experiencing pathologic pain would warrant concern. Given that CBD also modulated some cytokines in healthy rats, it will be important to determine whether these effects are deleterious, particularly if they persist during long-term use.

CBD increased IL- 6 but decreased IL- $1 \beta$ and IL-10 in the present study. No previous studies have investigated CBD effects on these cytokines to our knowledge. Further studies are needed to characterize CBD and THC effects on inflammatory cytokines, including cytokines beyond those we investigated. It would be pertinent to characterize sex differences in CFA- and cannabinoid-induced changes in immune function within 48 hours of CFA injection, since that is when acute phase response proteins peak (Haschek et al., 2013).

Surprisingly, THC was antinociceptive but had limited effects on cytokines, whereas CBD produced minimal antinociception but had robust effects on cytokines. These results suggest that THC's antinociceptive effects in the CFA model are primarily due to its neural actions (Starowicz and Finn, 2017) rather than to its actions on the immune system. Further studies of CBD's immunomodulatory effect could clarify the utility of CBD for the treatment of autoimmune diseases.

In summary, the current study provides further evidence in support of medicinal cannabis use, specifically THC, for the treatment of chronic inflammatory pain. The present findings suggest that THC maintains its pain-relieving efficacy with short-term, repeated administration, with limited effects on cytokines. In contrast, CBD alone may provide weaker analgesia in chronic inflammatory pain states while substantially altering immune function. Given our findings of distinct and possibly complementary patterns of THC and CBD effect in the CFA model, in combination with recent reports of synergy between THC and CBD in neuropathic pain models (Casey et al., 2017; King et al., 2017), it will be important to determine whether THC-CBD combinations provide more optimal analgesia than either drug alone under conditions of pathologic inflammation.

\section{Acknowledgments}

The authors thank H. Gogulski and K. Hewitt for excellent technical assistance.

\section{Authorship Contributions}

Participated in research design: Britch, Goodman, Craft.

Conducted experiments: Britch, Pondelick.

Performed data analysis: Britch, Pondelick.

Wrote or contributed to the writing of the manuscript: Britch, Goodman, Wiley, Pondelick, Craft.

\section{References}

Baron EP, Lucas P, Eades J, and Hogue O (2018) Patterns of medicinal cannabis use, strain analysis, and substitution effect among patients with migraine, headache, arthritis, and chronic pain in a medicinal cannabis cohort. J Headache Pain 19:37. Bass CE and Martin BR (2000) Time course for the induction and maintenance of tolerance to $\Delta(9)$-tetrahydrocannabinol in mice. Drug Alcohol Depend 60:113-119.

Blyth FM, March LM, Brnabic AJM, Jorm LR, Williamson M, and Cousins MJ (2001) Chronic pain in Australia: a prevalence study. Pain 89:127-134

Booker L, Naidu PS, Razdan RK, Mahadevan A, and Lichtman AH (2009) Evaluation of prevalent phytocannabinoids in the acetic acid model of visceral nociception. Drug Alcohol Depend 105:42-47.

Britch SC, Wiley JL, Yu Z, Clowers BH, and Craft RM (2017) Cannabidiol- $\Delta^{9}$-tetrahydrocannabinol interactions on acute pain and locomotor activity. Drug Alcohol Depend 175:187-197.

Bronte V and Pittet MJ (2013) The spleen in local and systemic regulation of immunity. Immunity 39:806-818.

Buchweitz JP, Karmaus PWF, Harkema JR, Williams KJ, and Kaminski NE (2007) Modulation of airway responses to influenza A/PR/8/34 by delta9tetrahydrocannabinol in C57BL/6 mice. J Pharmacol Exp Ther 323:675-683.

Casey SL, Atwal N, and Vaughan CW (2017) Cannabis constituent synergy in a mouse neuropathic pain model. Pain 158:2452-2460.

Cooper ZD and Haney M (2014) Investigation of sex-dependent effects of cannabis in daily cannabis smokers. Drug Alcohol Depend 136:85-91.

Cooper ZD and Haney M (2016) Sex-dependent effects of cannabis-induced analgesia. Drug Alcohol Depend 167:112-120.

Costa B, Colleoni M, Conti S, Parolaro D, Franke C, Trovato AE, and Giagnoni G (2004) Oral anti-inflammatory activity of cannabidiol, a non-psychoactive constituent of cannabis, in acute carrageenan-induced inflammation in the rat paw. Naunyn Schmiedebergs Arch Pharmacol 369:294-299.

Costa B, Trovato AE, Comelli F, Giagnoni G, and Colleoni M (2007) The nonpsychoactive cannabis constituent cannabidiol is an orally effective therapeutic agent in rat chronic inflammatory and neuropathic pain. Eur $J$ Pharmacol 556: 75-83.

Cox ML and Welch SP (2004) The antinociceptive effect of Delta9-tetrahydrocannabinol in the arthritic rat. Eur J Pharmacol 493:65-74.

Craft RM, Kandasamy R, and Davis SM (2013) Sex differences in anti-allodynic, antihyperalgesic and anti-edema effects of $\Delta(9)$-tetrahydrocannabinol in the rat. Pain 154:1709-1717.

Craft RM, Wakley AA, Tsutsui KT, and Laggart JD (2012) Sex differences in cannabinoid 1 vs. cannabinoid 2 receptor-selective antagonism of antinociception produced by delta9-tetrahydrocannabinol and CP55,940 in the rat. J Pharmacol Exp Ther 340:787-800.

Faul F, Erdfelder E, Lang A-G, and Buchner A (2007) G*Power 3: a flexible statistical power analysis program for the social, behavioral, and biomedical sciences. Behav Res Methods 39:175-191.

Galiègue S, Mary S, Marchand J, Dussossoy D, Carrière D, Carayon P, Bouaboula M, Shire D, Le Fur G, and Casellas P (1995) Expression of central and peripheral cannabinoid receptors in human immune tissues and leukocyte subpopulations. Eur J Biochem 232:54-61.

Gallily R, Yekhtin Z, and Hanuš LO (2015) Overcoming the bell-shaped doseresponse of Cannabidiol by using Cannabis extract enriched in Cannabidiol. Pharmacol Pharm 06:75-85.

Gallily R, Yekhtin Z, and Hanuš LO (2018) The anti-inflammatory properties of terpenoids from Cannabis. Cannabis Cannabinoid Res 3:282-290.

Gleicher N and Barad DH (2007) Gender as risk factor for autoimmune diseases. $J$ Autoimmun 28:1-6.

Greene NZ, Wiley JL, Yu Z, Clowers BH, and Craft RM (2018) Cannabidiol modulation of antinociceptive tolerance to $\Delta^{9}$-tetrahydrocannabinol. Psychopharmacology (Berl) 235:3289-3302.

Grubbs F (1969) Procedures for detecting outlying observations in samples. Technometrics 11:1-21.

Guerrero-Alba R, Barragán-Iglesias P, González-Hernández A, Valdez-Moráles EE Granados-Soto V, Condés-Lara M, Rodríguez MG, and Marichal-Cancino BA (2019) 
Some prospective alternatives for treating pain: the endocannabinoid system and its putative receptors GPR18 and GPR55. Front Pharmacol 9:1496.

Hammell DC, Zhang LP, Ma F, Abshire SM, McIlwrath SL, Stinchcomb AL and Westlund KN (2016) Transdermal cannabidiol reduces inflammation and painrelated behaviours in a rat model of arthritis. Eur J Pain 20:936-948.

Hanuš LO, Meyer SM, Muñoz E, Taglialatela-Scafati O, and Appendino G (2016) Phytocannabinoids: a unified critical inventory. Nat Prod Rep 33:1357-1392.

Haschek WM, Rousseaux CG, and Wallig MA (2013) Toxicologic pathology: an introduction, in Haschek and Rousseaux's Handbook of Toxicologic Pathology, 3rd ed. (Haschek WM, Rousseaux CG, and Wallig MA eds) pp 1-9, Academic Press, Boston.

Jamontt JM, Molleman A, Pertwee RG, and Parsons ME (2010) The effects of $\Delta$-tetrahydrocannabinol and cannabidiol alone and in combination on damage, inflammation and in vitro motility disturbances in rat colitis. $\mathrm{Br} J$ Pharmacol 160 $712-723$.

Katchan V, David P, and Shoenfeld Y (2016) Cannabinoids and autoimmune diseases: a systematic review. Autoimmun Rev 15:513-528.

King KM, Myers AM, Soroka-Monzo AJ, Tuma RF, Tallarida RJ, Walker EA, and Ward SJ (2017) Single and combined effects of $\Delta^{9}$-tetrahydrocannabinol and cannabidiol in a mouse model of chemotherapy-induced neuropathic pain. $\mathrm{Br}$ $J$ Pharmacol 174:2832-2841.

Klein SL and Flanagan KL (2016) Sex differences in immune responses. Nat Rev Immunol 16:626-638.

Klein TW and Cabral GA (2006) Cannabinoid-induced immune suppression and modulation of antigen-presenting cells. J Neuroimmune Pharmacol 1:50-64.

Kong WL, Peng YY, and Peng BW (2017) Modulation of neuroinflammation: role and therapeutic potential of TRPV1 in the neuro-immune axis. Brain Behav Immun 64 354-366.

Lefevre N, Corazza F, Duchateau J, Desir J, and Casimir G (2012) Sex differences in inflammatory cytokines and CD99 expression following in vitro lipopolysaccharide stimulation. Shock 38:37-42.

Malfait AM, Gallily R, Sumariwalla PF, Malik AS, Andreakos E, Mechoulam R, and Feldmann M (2000) The nonpsychoactive cannabis constituent cannabidiol is an oral anti-arthritic therapeutic in murine collagen-induced arthritis. Proc Natl Acad Sci USA 97:9561-9566.

McKinney DL, Cassidy MP, Collier LM, Martin BR, Wiley JL, Selley DE, and SimSelley LJ (2008) Dose-related differences in the regional pattern of cannabinoid receptor adaptation and in vivo tolerance development to delta9tetrahydrocannabinol. $J$ Pharmacol Exp Ther 324:664-673.

Mechoulam R, McCallum NK, and Burstein S (1976) Recent advances in the chemistry and biochemistry of cannabis. Chem Rev 76:75-112.

Moudgil KD and Choubey D (2011) Cytokines in autoimmunity: role in induction, regulation, and treatment. $J$ Interferon Cytokine Res 31:695-703.

Moxley G, Posthuma D, Carlson P, Estrada E, Han J, Benson LL, and Neale MC (2002) Sexual dimorphism in innate immunity. Arthritis Rheum 46:250-258.

Puffenbarger RA, Boothe AC, and Cabral GA (2000) Cannabinoids inhibit LPSinducible cytokine mRNA expression in rat microglial cells. Glia 29:58-69.

Raoof R, Willemen HLDM, and Eijkelkamp N (2018) Divergent roles of immune cells and their mediators in pain. Rheumatology (Oxford) 57:429-440.
Redmond WJ, Goffaux P, Potvin S, and Marchand S (2008) Analgesic and antihyperalgesic effects of nabilone on experimental heat pain. Curr Med Res Opin 24: $1017-1024$

Rock EM, Limebeer CL, and Parker LA (2018) Effect of cannabidiolic acid and $\Delta^{9}$ tetrahydrocannabinol on carrageenan-induced hyperalgesia and edema in a rodent model of inflammatory pain. Psychopharmacology (Berl) 235:3259-3271.

Sanders J, Jackson DM, and Starmer GA (1979) Interactions among the cannabinoids in the antagonism of the abdominal constriction response in the mouse. Psychopharmacology (Berl) 61:281-285.

Smith S, Terminelli C, and Denhardt G (2000) Effects of Cannabinoid receptor agonist and antagonist ligands on production of inflammatory cytokines and antiinflammatory interleukin-10 in endotoxemic mice. $J$ Pharmacol Exp Ther 293: 136-150.

Sofia RD, Vassar HB, and Knobloch LC (1975) Comparative analgesic activity of various naturally occurring cannabinoids in mice and rats. Psychopharmacology (Berl) 40:285-295.

Starowicz K and Finn DP (2017) Cannabinoids and pain: sites and mechanisms of action. Adv Pharmacol 80:437-475.

Stockings E, Campbell G, Hall WD, Nielsen S, Zagic D, Rahman R, Murnion B, Farrell M, Weier M, and Degenhardt L (2018) Cannabis and cannabinoids for the treatment of people with chronic noncancer pain conditions: a systematic review and meta-analysis of controlled and observational studies. Pain 159:1932-1954.

Tighe PJ, Riley JL III, and Fillingim RB (2014) Sex differences in the incidence of severe pain events following surgery: a review of 333,000 pain scores. Pain Med 15: 1390-1404.

Tseng AH and Craft RM (2001) Sex differences in antinociceptive and motoric effects of cannabinoids. Eur J Pharmacol 430:41-47.

Unruh AM (1996) Gender variations in clinical pain experience. Pain 65:123-167.

Varvel SA, Wiley JL, Yang R, Bridgen DT, Long K, Lichtman AH, and Martin BR (2006) Interactions between THC and cannabidiol in mouse models of cannabinoid activity. Psychopharmacology (Berl) 186:226-234.

Wakley AA, Wiley JL, and Craft RM (2014) Sex differences in antinociceptive tolerance to delta-9-tetrahydrocannabinol in the rat. Drug Alcohol Depend 143:22-28.

Wakley AA, Wiley JL, and Craft RM (2015) Gonadal hormones do not alter the development of antinociceptive tolerance to delta-9-tetrahydrocannabinol in adult rats. Pharmacol Biochem Behav 133:111-121.

Wiley JL, O'connell MM, Tokarz ME, and Wright MJ Jr (2007) Pharmacological effects of acute and repeated administration of Delta(9)-tetrahydrocannabinol in adolescent and adult rats. $J$ Pharmacol Exp Ther 320:1097-1105.

Yee JR and Prendergast BJ (2010) Sex-specific social regulation of inflammatory responses and sickness behaviors. Brain Behav Immun 24:942-951.

Zandman-Goddard G, Peeva E, and Shoenfeld Y (2007) Gender and autoimmunity. Autoimmun Rev 6:366-372.

Address correspondence to: Rebecca M. Craft, Department of Psychology, Washington State University, Johnson Tower 233, Pullman, WA. E-mail: Craft@wsu.edu 